\title{
Extent of Arabic Language Teachers' Awareness of Reflective Thinking Skills and Relation to Students' Attitude toward Arabic Language Course at Secondary Stage in Saudi Arabia
}

\author{
Haifa Awwad Alhawamdeh (Corresponding author) \\ Department of Curriculum and Instruction, College of Education \\ Najran University, Saudi Arabia
}

Received: February 19, 2017 Accepted: March 7, 2017 Published: March 31, 2017

doi:10.5296/ire.v5i1.11023 URL: http://dx.doi.org/10.5296/ire.v5i1.11023

\begin{abstract}
The current study aims to indicate the extent to which Arabic language teachers are aware of reflective thinking skills and their relationship to their students' attitude toward the course of Arabic language at the secondary stage in the Kingdom of Saudi Arabia. The study adopted the survey descriptive method. The population consisted of all students enrolled in the secondary schools of Najran (546) and (46) teachers, the sample was randomly selected numbered (40) teachers. The author used the questionnaire as a tool for data collection. The study showed that the students were able to master well the skills of reflective thinking, where they were able to retain and transfer reflective thinking in positions similar to those positions they trained. According to the study findings, some recommendations have been made.
\end{abstract}

Keywords: Arabic language teachers, reflective thinking skills, students' attitudes

\section{Introduction}

Recent developments in the educational system require teachers to reshape their knowledge and beliefs about teaching and learning continuously. Such knowledge has become an integral part of teacher preparation programs. Searching for skills that enable students to be creative and unique is the responsibility of teachers who take over teaching and learning. So, they assume the responsibility for improving their teaching practices continuously. Because the emerging attitudes in education which directed educators to be concerned with all forms 
of learning skills that believe that learner builds knowledge through interaction and integration with the educational content and the surrounding environment, and considering reflection a key factor in the processes of teaching and learning, are of the factors that contribute to focus on reflective thinking skills in the educational field (Farrell, 2008). Reflective thoughts, involving their practical and theoretical implications, strongly affected the educational scene. This is at the educators and authors increasing conviction with the importance of these thoughts. Reflective practices are generally essential aspects of the educators' professional development (Kim, 2005). They also improve teachers' performance. Because these ideas agree with the international standards related to preparing mathematics teachers and focusing on their role as members in the field, communicators with learners and their families, and planners and facilitators of learning, research activity expanded (NCTM, 2003).

Over the past two decades, countries focused on the development of thinking skills as they positively affect the life of human community. Educational policies began to focus on the development of children's thinking skills to be the basis of outstanding thinkers; such policies are an important means of develop a high level of self-awareness for self-development. The teaching of thinking cannot be simply described as delivering or transferring certain skills, but motivating the attitudes of exploration, investigation, curiosity, and the analysis of convictions and beliefs interrelated within sub-consciousness. A successful community is that of thinking where members accomplish lifelong learning through observation, analysis, evaluation, experimentation and problem solving. This focuses on the effort exerted to improve and develop the quality of education.

Reflection and reflective thinking are one of the sustainable development tools for teachers as they help them do professional practices, give them a higher level of insight, and deepen thinking about performance and behavior. They develop, changes, and improve behavior. By using multiple tools of reflective thinking, e.g. diaries, short story records, check lists, newspapers of reflection, taking notes in a systematic and organized way, and participation in reflection conversations with others and self-reflection. Accordingly, a person realizes the level of performance.

Mohamed (1993) believes that reflective thinking is one of the most significant mental and cognitive core processes that distinguish students in the speed of response to the different life situations, and their competence and accuracy in providing appropriate responses. Reflective students often tend to take time in responding, and take a longer time in the allocation of the alternatives available to them before making decisions about their implementation. Reflective thinking is an important educational aim in classroom teaching. Thus, a teacher should focus on this kind of thinking because of its great benefit to the students as it is a key objective to the various global educational policies.

According to Ibrahim (2005), in reflective thinking "a student reflects on the situation in hand, analyzing it to its elements and puts the necessary plans to understand. Then, the desired results are reached". Anzai and Paik (2000) claimed that the fear of interaction or oral communication with others is a problem for many students because it negatively affects 
students' attitudes towards learning, motivating them to avoid participation in social and educational activities. These in turn affect their academic performance.

Reflective thinking is essential for students because it allows them to rethink of, scrutinize, and study the idea from various aspects. It also explores the relations among these elements and reveals their gaps. It also finds out the reasons that lead to these results through links that connect the elements and provides solutions to the problems. Accordingly, a person is capable of learning by himself; the objective of modern education.

The development of reflective thinking is one of the key objectives of teaching because it motivates the student to plan and evaluate his approach in the processes and steps to be followed in taking a decision. It is based on student's reflection on information that in turn affects the learner's mind. This provides a meaningful learning; the focus of modern learning strategies (Al kawsma \& Mohamed, 2013).

Despite the attempts of educators to classify characteristics and features in one way or another, they cannot be separated. They are integrated. It has been pointed out that social characteristics affect cognitive and emotional features that each of them affects and is affected by others. Together, they form the learner's personality. They also generate certain motives and desires towards learning, affecting, in turn, the attitudes towards this learning. Despite the common features among adults, each of them has shared and personal ones that determine a special attitude of the learning process. Therefore, it is not enough to measure one's attitude towards learning, but a broad sample of attitudes should be measured to be able to define attitudes, in general.

\subsection{Statement of the Problem}

Educational institutions in Saudi Arabia seek to develop thinking among students, as one of the main objectives of the educational process in the twenty-first century. Reflective thinking skills are an important aspect of the student's life, in general, because it effectively contributes in the modernization and development of the educational process. The aim is to provide teaching and learning methods that achieve the objective of teaching the different strategies to students, e.g. reflective thinking and attitudes. The variables include competence in speaking and interacting with others, moving from traditional education to that one that promotes student's creativity and excellence.

\subsubsection{Study Questions}

The problem of the current study can be defined in answering the following major question:

To what extent are Arabic language teachers aware of reflective thinking skills and their relationship to their students' attitude towards the course of Arabic language at secondary stage in Kingdom of Saudi Arabia?

In addition, the following minor questions are answered:

1) To what extent do students of Arabic language in Najran secondary schools have reflective thinking skills? 
2) Are there statistically significant differences in the level of reflective thinking skills among Arabic language's students in Najran secondary schools to be attributed to their attitudes at the level of $(\alpha \leq 0.05)$ ?

3) Are there statistically significant differences in the level of reflective thinking skills among Arabic language's students in Najran secondary schools to be attributed to their educational level at the level of $(\alpha \leq 0.05)$ ?

4) Are there statistically significant differences in the level of reflective thinking skills among Arabic language's students in Najran secondary schools to be attributed to the course at the level of $(\alpha \leq 0.05)$ ?

\subsection{Objectives}

The current study aims to:

1. Identify the extent to which Arabic language teachers are aware of reflective thinking skills and their relationship to their students' attitude towards the course of Arabic language at the secondary stage in the Kingdom of Saudi Arabia.

2. Identify extent of reflective thinking that Arabic language students at the secondary stage in Najran schools have due to students ' attitudes.

\subsection{Limitations}

The study is limited to second secondary stage students in Najran Educational Area for the first semester 2016/2017

\subsection{Definition of Terms}

Based on the importance of this topic, it is necessary to identify its concept in the related educational literature. There are many definitions of reflection and reflective thinking, in general. However, there is not a clear-cut definition because of the multiplicity of perspectives and intellectual schools. Rather, there is a common denominator among all of them, i.e. reflection. In addition, reflective thinking has become an essential feature for the teacher.

\subsubsection{Reflective thinking}

It is the ability to vigilantly handle events, situations and educational stimuli, analyzing them profoundly and carefully to take the right decision at the right time and place to achieve the expected objectives (Barakat, 2005). Procedurally, reflective thinking is "the total degree obtained by the participant in reflective thinking test on Eysenck \& Weilson Reflectiveness Scale. It refers to "the purposeful mental activity which is based on reflection through visual skills, revealing fallacies, reaching conclusions, giving satisfactory explanations, and providing proposed solutions to scientific problems" (Al Qatrawy, 2010) Abdulwahab (2005) defines it as "the ability of foresight, perceiving relationships, making use of the facts to 
identify and support the learner's perspective, reviewing alternatives, and taking actions appropriate for the situation".

The author selected the second secondary stage because thinking skills moderately require intellectual maturity that these students have. They refuse to take things for granted, unlike the previous stage where students receive information without discussion.

\section{Theoretical Framework}

\subsection{The concept of Thinking}

Thinking is a complicated concept that involves interrelated aspects and components that reflect the complex nature of the brain. It is a series of activities of the brain when a stimulus is received by one or more of the five senses. It is an abstract concept that involves invisible and intangible activities. What are observed are the outcomes of thinking, whether written, verbal, dynamic, or visual (Shwaheen, 2009).

Thinking is a blessing that has been bestowed on the human being. It occupied many authors, educators, and philosophers for ages. All the philosophical, intellectual, psychological, and educational schools were interested in the development of student ' intellect and thinking to become more capable of face difficulties and problems, either in the academic fields or the social, economic, educational, moral, and other aspects of life (Al atom, 2009). Reflective thinking is an educational necessity because of its benefits, as follows:

It helps students think deeply, explore new educational mechanisms, reflect on multiple ideas about the subject, and evaluate work by themselves. This is based on consolidating students' perspectives by helping them solve problems and accurately analyze things to develop their psychological aspect (Khwalda, 2012). It also helps the teacher achieve a better understanding of students' learning types and use a variety of teaching methods. It works on improving the teaching methods and professionally practice responsibilities. The author believes that the relationship between reflective thinking and Arabic language is deeply rooted because of the meanings of the Arabic language that allow the reader and listener to reflect instead of taking them as granted. In order to express in a certain situation or an incident, or write on a subject, recalling and organizing previous knowledge. Accordingly, conclusions and solutions are reached. Then, the solutions are applied. These are the stages of reflective thinking. Teaching for thinking development is an idea that moved from theory to practice because thinking is a main educational aim that helps achieve the course's aim and helps teacher in memorization by preparing the student in an environment of psychological security to enhance their thinking ability. There are many opinions regarding the classification of reflective thinking skills, such as Langer and Colton who classified thinking according to identifying, reframing and organizing the problem to find alternative solutions through the proposed actual experience to make alternative decisions. Halton and Smith offered another classification where the problem is defined according to describing a certain action, defining the potential causes, and interpreting available data. 


\subsection{The Concept of Attitudes}

\section{Anastasi' definition}

An attitude is often known as a tendency to respond positively or negatively towards a certain set of stimuli.

\section{Thurstone ' definition}

The psychological attitude is a generalization of the person's responses to make the behavior near or far from a certain concept.

\section{Boring \& Langfield ' definition}

The attitude is the mental, emotional and, cognitive condition about certain aspects of the surrounding environment.

\section{The definition of Krech et al}

The attitude is a continuous planning of the emotional and cognitive processes about some aspects of the surrounding environment.

\section{Guilford's definition}

The attitude is a specific or general readiness in the individuals but it is somehow acquired and motivates them to respond to things and situations, whether with or against.

\section{Allport's definition}

The attitude a mental and neural state of readiness by experience and has a dynamic or directed effect on the individual's responses to all related topics and positions.

Abdel Rahim (1988) concludes that the attitude, as a psychological phenomenon, is a willingness or tendency to respond in a certain way to certain situations or stimuli. Such willingness, either permanent or temporary, is formed by experience as a result of the person's interaction with environment and guides the person for situations and things; the subject of the attitude. Zahran (1974) believes that "the attitude is a hypothetical composition or latent variable (between the stimulus and response). It is a psychological willingness or a learned mental and neural of the positive or negative response towards persons, objects, topics, situations, or symbols in the environment that evoke this response'. Accordingly, attitudes include three basic components: the cognitive, emotional and, behavioral components that are totally integrated. The cognitive component is essential for creating feelings, altitudes, desires, and situations as it cannot be generated without being aware of the situation, incident or the object that the person faces. A learner who experience learning and teaching has certain feelings and attitudes (negative or positive), depending on experience. They can be measured and defined by authentic tools. Attitudes represent the peak of the emotional aspect because they motivate a person to take a situation of acceptance or rejection towards a certain topic. They are the total of knowledge's impact on feelings, desires, tendencies, and all components of the emotional aspect. Hence, they are defined as situations of acceptance or refusal towards a certain topic or issue. They also give a meaning on the 
individual's perception and activities that help accomplish objectives. In addition, the study of attitudes is one of the most important items of changing human behavior to respond to stimuli that form negative attitudes (Olaimat, 1994).

Attitudes that form the basis of our study and are acquired by learners are not formed in vacuum because the development of emotional aspects occurs within the cognitive and psychodynamic changes and developments that are integral. Getting knowledge and the learner's feeling of its importance in life, clarity, and presentation positively contribute to the development of attitudes towards learning, but learner's inability to learn may cause frustration and generate negative attitudes to renounce learning. Therefore, to ensure having the positive attitudes, the most appropriate conditions that promote the motives and attitudes toward learning. Aboalhob (1981) refers to "the importance of having negative attitudes as a result of the emotional problems that face the adult learner, e.g. tension, anxiety, sense of inferiority, shyness, timidity, inability to assume responsibility and lack of self-confidence.

Many studies confirm that the attitude is not a simple component, but a compound one that covers several elements. After reviewing literature, it became clear that authors agree that most appropriate statistical method to measure attitudes is using Likert scale, where responses to each paragraph are distributed on the 5-item scale which reflects attitude's severity (i.e., negative, positive, or neutral) (Shaw \& Wright, 1967) . Accordingly, the attitudes of the participant are estimated, in general, by calculating the points of all paragraphs and estimating the general mean by dividing the total mark on the number of the questionnaire's items.

Hence, theoretical framework of the study is concluded, as follows:

1) Reflective thinking among the second secondary stage students has a special position in the educational system, either vertically or horizontally, as the base to begin comprehensive and sustainable programs for learning and human resources development, in general.

2) Attitudes have great importance in achieving the desired objectives of teaching Arabic language, particularly regarding attracting the largest number of students; they are key factors in increasing learners' realism towards learning.

3) Attitudes are measurable and educational literature is full of many scientific measurements. However, the Arab region really needs such measurements in general because of neglecting on the part of authors in the field of Arabic language teaching.

4) There are many methods of attitudes' measurement, the most accurate, appropriate, and widely used and for second secondary stage students who study Arabic language is Likert scale.

Psychologically, an attitude is a condition of mental and neural readiness that makes the individual respond to stimuli related to a certain topic or situation in a certain way. It is a personal component indicated by external responses and can be acquired by experiencing and interacting with several situations. It is an emotional aim that the educational institution, with 
all structure and levels, to be achieved among learners because it psychologically affects behaviors and responses to the various inputs of the educational process, e.g. curriculum, activities, classroom environment, teachers, peers and students themselves. This, in turn, affects students to accomplish the assigned tasks. It also facilitates adjustment to school environment, in particular and social life, in general (Nashawati, 1998).

\section{Literature Review}

Numerous studies and researches were conducted on reflective thinking locally, regionally, or globally level have been adopted, focusing on several fields, such as student's level of reflective thinking, strategies and methods of thinking development, the effectiveness of reflective thinking in other variables, school and university.

Afana \& Iulu (2002) aimed to determine the level of reflective thinking skills in the problems of field teaching among students at the College of education, Islamic University in Gaza. The study sample comprised (400) male and female students. It concluded that there are statistically significant differences in reflective thinking between the two groups of Sciences and their peers of English, in favor of Sciences' students. In addition, students did not master reflective thinking. Barakat (2005) aimed to identify the relationship between reflective thinking and achievement among a sample of university and high school students according to some variables. The study covered (400) students, equally males and females. Ysenck \& Weilson Reflectiveness Scale was applied. Results indicated that there were statistically significant differences on the level of reflective thinking that could be attributed to the academic level. In addition, there were not statistically significant differences on the level of reflective thinking that could be attributed to gender or achievement. Abdulwahab (2005) aimed to determine the effectiveness of using metacognition strategies in physics achievement and the development of reflective thinking and the tendency to use them among second Al-Azhar secondary school. The experimental approach was used. The sample comprised the students of the second year of Banha secondary institute for males in the second semester. Results indicated that students of the empirical group excel in the development of reflective thinking skills. Shakaa (2007) aimed to identify reflective thinking level among undergraduate and postgraduate students at Al-Najah University, Palestine and to determine differences in levels according to college, gender, and academic level. The study comprised (641) female and male students; (550) of undergraduate students and (91) of master students. Ysenck \& Weilson Reflectiveness Scale was applied. Results indicated that reflective thinking level of Al-Najah university students was good. They also reported that there were statistically significant differences in the level of reflective thinking attributed to college, in favor of Colleges of Humanities. While there were statistically significant differences attributed to the impact of academic level and in favor of Master's students, there were no statistically significant differences attributed to gender.

Ogonor and Badmus (2006) investigated the impact of reflective thinking on student teacher's beliefs towards their future roles. Therefore, it was applied to a sample of (304) male and female students in the final year of Education at a Nigerian university. Data were collected using a questionnaire of open-ended questions in various fields. Results suggested 
that student teachers' beliefs of reflective teaching influenced on their current and previous knowledge in education, their professional development and experience. Most of the participants reported that reflective thinking motivates them on excellence. Scott and Isa (2007) conducted a case study that aimed to investigate the relation between teachers' reflective practices, students' satisfaction, and learning outcomes. The study covered (45) students. Several tools were used to collect data, such as: questionnaire, interviews with students and teachers ' blogs. It concluded that there was a significant relation between reflective practices and of achievement. It also raised student's satisfaction and responses all over the year. Furthermore, outcomes' quality progressively enhanced, supported sound teaching, and contributed to the development of teacher's cognitive challenges in evaluation. Ellison (2008) addressed the effectiveness of a reflective teaching- based training program teaching for basic school teachers. It was applied to (6) teachers, who were divided into two groups; (3) teachers of mathematics and (3) teachers of science. Data were collected using classroom observation card. Results showed that there were positive outcomes of applying this program to teachers' classroom practices, such as: participatory integration, critical reflective dialogue, and curriculum development; accordingly, learning outcomes were positively affected through integration in classroom activities and cooperative discussions. Vanhoof et al. (2006) conducted study over five years that aimed to identify university students' attitudes towards Statistics (attitude towards Statistics field, attitude towards Statistics course) and their relation to exams' results. The study was applied to (264) students in the educational science of Statistics course. Results illustrated that there was a statistically significant positive relation between students' attitude towards Statistics and achievement in the first year. In addition, there was a positive relation between students' attitude towards the use of Statistics in their specialization and their scores in the graduation project. However, there was not a statistically significant positive relation between students' attitude towards Statistics and their achievement in the general exams as it was only limited to the result of Statistics exam. It could be concluded that there is a shortage of Arab studies that addressed students' reflective thinking and attitudes towards Arabic language as it was limited to foreign studies. In addition, there were not studies that addressed reflective thinking and students attitudes from teachers' perspective in Saudi Arabia, to the author's knowledge. She benefited from literature in formulating the statement of the problem, discussing results and the adopted scales.

\section{Methodology}

\subsection{Method}

The present study adopted the survey descriptive approach. Its population comprised all students enrolled in regular study in Najran secondary stage, numbered (546) students and (46). A sample of (40) teachers was randomly selected sample to whom the questionnaire was distributed to all, but 4 questionnaires were excluded because of invalidity.

\subsection{Tool's validity}

The questionnaire, in its primary form, was submitted to a number of university specialized professors. They expressed their views and made notes on the reliability of the 
questionnaire's items and their correlation to its aspects. Accordingly, some items were excluded and others were modified.

The following tools were used:

\section{1) Ysnek \& Weilson Reflectiveness Scale}

Barakat (2005) translated the scale that consisted of (30) items to which responses are (agree and disagree). In total, (20) items represent a positive attitude to reflective thinking and the student is graded (1) for (agreement) and (0) for (disagreement). The other (10) items represent a negative attitude to reflective thinking, and the participant is awarded a mark for (disagreement) and (0) for (agreement). Hence, the total scores on the scale ranges from (0) to (30). This scale has high degrees of validity on Pearson correlation coefficient (from 0.94 to 0.73 ) which is statistically significant at the level of (0.05). After applying the scale to a pilot sample of (32) university students after period of (3) weeks, reliability coefficient were from $(0.83)$ to $(0.91)$ and the total reliability coefficient was $(0.86)$. To determine the tool's validity and reliability, it was submitted to a group of reviewers, the required modifications to the items were made, and the reliability of reflective thinking test was estimated. Then, the scale was applied to a pilot representative sample and reliability coefficient rated ( 0.80 to $0.84)$ while the total reliability coefficient was (0.82).

\section{Test scoring:}

Reflective thinking level is measured, as follows:

- Less than 10 degrees; Weak

- $\quad$ (10) to (19) degrees; Medium

- (30) to (20); Good

2) Student's attitudes test

This study used students' attitudes scale. Ali (2005) translated the scale into Arabic. It was prepared by McDowell (2000). It consisted of (21) five-items. Its reliability was tested using Cronbach's alpha, where reliability coefficient was (0.748). Using spilt half method, reliability coefficient was $(0.466)$. They are both statistically significances at the level of (0.01).

\section{Scale's Validity:}

Validity of the scale is determined by criterion-related validity where validity coefficient rated $(0.186-0.460)$. It is statistically significant at the levels of $(0.01)$ and $(0.05)$.

Correction of the scale:

The test consists of (21) items and the total degree is (105). If students obtain $(73 \%$ and more), their attitudes are high. If students obtain (27\% and less), their attitudes are low. If students obtain $(27 \%-73 \%)$, their attitudes are medium. 


\section{Results and Discussion}

Arithmetic mean, standard deviation, range, and the percentage of reflective thinking of Najran secondary schools' students were estimated to answer the first question, as shown in Table (1).

Table (1). Arithmetic mean, standard deviation, extent and the percentage of reflective thinking

\begin{tabular}{cccc}
\hline Arithmetic mean & Standard deviations & Extent & Percentage \\
\hline \multirow{2}{*}{19.0395} & 5.54262 & $27-7$ & $66 \%$ \\
& & 13 & \\
\hline
\end{tabular}

Table (1) shows that the level of reflective thinking of Najran secondary schools students was medium, with an arithmetic mean of (19.0395), a standard deviation of (5.54262), and a percentage of $(66 \%)$. The lowest score obtained was (7). Therefore, frequencies and percentage were estimated according to the levels of reflective thinking and the sample of the study.

Table (2). Frequencies and percentages of the levels of reflective thinking among the students of Najran secondary schools

\begin{tabular}{lllllll}
\hline \multirow{2}{*}{ Level } & \multicolumn{2}{l}{ Distinguished } & Normal & & \multicolumn{2}{l}{ Distinguished/ Normal } \\
\cline { 2 - 7 } & Frequency & Percentage & Frequency & Percentage & Frequency & Percentage \\
\hline Good & 100 & $39 \%$ & 16 & $6 \%$ & 116 & $46 \%$ \\
Medium & 20 & $8 \%$ & 104 & $41 \%$ & 124 & $49 \%$ \\
Weak & - & - & 13 & $5 \%$ & 13 & $5 \%$ \\
Total & 120 & $47 \%$ & 133 & $53 \%$ & 253 & $100 \%$ \\
\hline
\end{tabular}

Table (2) shows that (good level) rates (46\%), where distinguished students represent (39\%) and the normal represent (6\%). In addition, medium level rates (49\%), where the distinguished represent (8\%) and the normal represent (41\%). Weak is ranked last of $(5 \%)$, where the distinguished represent $(0 \%)$ and the ordinary represent $(5 \%)$.

This result is attributed to the fact that all students have reflective thinking, in varying degrees. It is developable and improvable in case of setting educational programs appropriate for students' mental and cognitive levels, mainly activating students' role in the educational process, giving them freedom to interact with each other by using a variety of educational methods. Here, distinguished and normal students are referred to as having been trained on reflective thinking skills through reflective thinking curriculum and learning which is essential for learning Arabic Language. However, they only achieve the medium level of reflective thinking.

Results of this study agree with Afana and Lulu (2002) indicating that the level of reflective thinking among secondary students is good, but they differ from Shakaa (2007) indicating that the reflective thinking is not mastered, and from Barakat (2004) reporting that the level 
of reflective thinking is good.

Results of the first sub question: In order to answer this question, arithmetic mean, standard deviation, range, and the percentage of the total degree of the attitudes of Najran secondary schools student, as shown in Table (3).

Table (3). Arithmetic mean, standard deviation, extent and the percentage of the total degree of students' attitudes scale

\begin{tabular}{lccc}
\hline Arithmetic mean & Standard deviation & Extent & Percentage \\
\hline 71.7510 & 19.59909 & $100-12$ & $34 \%$ \\
& & $(88)$ & \\
\hline
\end{tabular}

Table (3) shows that the attitudes of Najran secondary schools students were medium, with an arithmetic mean of (71.7510), a standard deviation of (19.59909) and a percentage of (34\%). The lowest degree obtained by students was (12). Therefore, frequencies and percentage were calculated according to students' attitudes and study sample (distinguished/normal) (as shown in table 4).

Table (4). Frequencies and percentages of the attitude of Najran secondary schools' students

\begin{tabular}{lllllll}
\hline \multirow{2}{*}{ Level } & \multicolumn{2}{c}{ Distinguished } & \multicolumn{2}{c}{ Normal } & \multicolumn{2}{c}{ Distinguished/ Normal } \\
\cline { 2 - 7 } & Frequency & Percentage & Frequency & Percentage & Frequency & Percentage \\
\hline High & 70 & $28 \%$ & 43 & $17 \%$ & 113 & $46 \%$ \\
Medium & 50 & $19 \%$ & 80 & $33 \%$ & 130 & $52 \%$ \\
Low & - & - & 10 & $3 \%$ & 10 & $3 \%$ \\
Total & 120 & $47 \%$ & 133 & $53 \%$ & 253 & $100 \%$ \\
\hline
\end{tabular}

The table shows that (high level) rate $(46 \%)$, where distinguished students represent $(28 \%)$ and the normal represent (17\%). In addition, medium level rates $(52 \%)$, where the distinguished represent (19\%) and the normal represent (33\%). Weak is ranked last of (3\%), where the distinguished represent $(0 \%)$ and the ordinary represent $(3 \%)$. This is attributed to the diversity of curricula which Najran secondary schools students study because they develop students' attitudes as they comprise scientific and human knowledge.

Herman (2002) indicated that studying Arabic language requires comprehensible thinking. Because language stresses logical sequence and using uniform teaching methods due to the nature of the preparatory year where the testing system is central as all students have the same tests and questions, teaching methods are similar. This motivates them to use developed educational methods, e.g. activities, brainstorming, and problem solving. Students are motivated to produce, not only learning, knowledge. These strategies are largely consistent with students' attitudes. This differs from Almeida (2002) indicating that student attitudes are negative because they prefer not to interact.

Results of the second sub question: To answer this question, arithmetic mean, standard deviations, and " $T$ " test are calculate for distinguished and normal students on students' 
attitudes scale (as shown in table 5).

Table (5). arithmetic mean, standard deviations and " $\mathrm{T}$ " test ( $\mathrm{T})$ of students' responses on reflective thinking scale

\begin{tabular}{|c|c|c|c|c|c|}
\hline Test & Sample & Arithmetic mean & Standard deviation & "T" value & $\begin{array}{l}\text { Statistical } \\
\text { significance }\end{array}$ \\
\hline Reflective & & 23.5750 & 2.77946 & \multirow{2}{*}{-20.755} & \multirow{2}{*}{.000} \\
\hline thinking & & 14.4917 & 3.90216 & & \\
\hline
\end{tabular}

Table (5) shows that there are statistically significant differences at a significance level of ( $\alpha$ $\leq 0.05$ ) between the means of students' responses in all fields and the total test in favor of distinguished students. This is because students were able to master reflective thinking skills as they were able to keep and transfer reflective thinking skills to situations similar to their training. Normal students obtained a medium level in reflective thinking because they mainly wrote the important information without paying attention to analysis, interpretation and conclusion, unlike those students who prefer to pay attention to the teacher because of the positive effects on recalling. Ibrahim (2005) reported that reflective thinking requires analyzing the situation into the different elements and searching for internal relations because it is used when feeling perplexed towards a problem or issue. This agrees with the literature, indicating that the training programs develop reflective thinking skills. This study differs from Barakat (2005) that reported the lack of statistically significant differences due to the impact of achievement on reflective thinking.

Results of the third sub question: To answer this question, arithmetic mean, standard deviations, and " $T$ " test are calculate for the responses of the distinguished and normal students on students' attitudes scale, as shown in table (5).

Table (6). Arithmetic mean, standard deviations and " $\mathrm{T}$ " test $(\mathrm{T})$ of students' responses on students' attitudes scale

\begin{tabular}{|c|c|c|c|c|c|}
\hline Test & Sample & Arithmetic mean & Standard deviation & $\begin{array}{l}\text { "T" } \\
\text { value }\end{array}$ & $\begin{array}{l}\text { Statistical } \\
\text { significance }\end{array}$ \\
\hline Cognitive & & 82.3667 & 13.37748 & \multirow{2}{*}{-9.966} & \multirow{2}{*}{.000} \\
\hline competence & & 63.4083 & 18.98850 & & \\
\hline
\end{tabular}

The table shows that there are statistically significant differences at a significance level of ( $\alpha$ $\leq 0.05$ ) between the means of students' responses in the total test. This is because students were able to master reflective thinking skills as they were able to keep and transfer reflective thinking skills to situations similar to their training. Normal students obtained a medium level in reflective thinking because they mainly wrote the important information without paying attention to analysis, interpretation and conclusion, unlike those students who prefer to pay attention to the teacher because of the positive effects on recalling.

This is acceptable because distinguished have motivation, curiosity, active interaction and 
positive participation, the ability to commit to the required tasks as they attend lectures and prepare before and after them, interacting with teachers, asking and evaluating mysterious questions to develop because of their multiple attitudes and focusing on their aims for academic excellence. They are also more confident and serious in ask about mysterious points in the course because of their sense of maturity and independence as these questions enhance their interactive competence. In addition, they are curious, fluent and often ask questions difficult to answer. This agrees with literature, indicating that distinguished students are less afraid of interaction than the normal ones. Results reported that achievement motivation and controlling academic activity are strongly related to students' attitudes. Furthermore, interaction is largely controlled by academic and cognitive aspects.

Results of the fourth sub question: To test the validity of this question, correlation coefficient was estimated between student degrees in reflective thinking, as shown in table (7).

Table (7). Results of Pearson correlation coefficient between students' attitudes and critical thinking

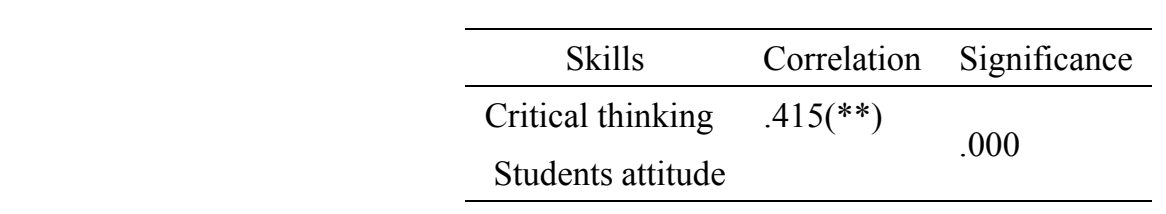

Significant at the level of 0.05 *

Table (7) shows that there is a positive relation between reflective thinking and students attitudes. It is statistically significant with correlation coefficient of $(0.415)$, referring to a direct relation; the more reflective thinking increased, the more attitudes students have.

This is attributed to the nature of curricula studied by Najran secondary schools' students, especially Arabic language, thinking skills, and learning because they allow students to discuss and interact positively and use mental styles to solve problems. It enables them to gather information from original sources by using scientific research methods, solving problems in the surrounding environment through brainstorming and criticizing ideas, issues, and finding appropriate solutions. Accordingly, these skills are an important indicator of the reflective thinker and students' attitudes.

\section{Recommendations}

- The need of the directorates of education to raise and develop students' attitudes competence and reflective thinking.

- Developing purposeful educational programs to increase reflective thinking level and cognitive competence to suit current trends.

- Conducting comprehensive studies to include large samples in all secondary schools in the Kingdom of Saudi Arabia.

\section{References}

AbdulRahim, T. (1988). Contemporary social psychology. Cairo: Egyptian National Library. 
Abdul, W., \& Fatima, M. (2005) the effectiveness of the use of certain strategies and metacognition in the achievement of Physics and the development of reflective thinking and the trend towards the use of second-Azhari secondary students The Scientific Journal of Education, Faculty of Education, Ain Shams University, 4(8).

Aboalhob. (1981). adult education and the challenges of the age. Continuing education, Volume III, the second year.

Afana \& Lulu. (2002). Reflective thinking levels of training field for student of Education College in Islamic university. Ain Shams University, scientific education Journal, 5(2).

Al kawsma \& Mohamed. (2013). The development of learning and thinking and research skills. Amman: Dar Al Safa for Publishing and Distribution, Amma.

Al Otom, A. (2009). Developing the skills of thinking about theoretical models and practical applications. Amman: almaysra for publishing and distribution.

Al Qatrawy, A. (2010). The effect of using strategy of similarities in the development of science processes and skills of reflective thinking in science among primary eighth-grade students. Unpublished MA Thesis, Islamic University of Gaza.

Almeida, E. (2002). A Discourse Analysis Of Student Perceptions Of There Communication Competence ",Paper Presented At The Annual Meeting Of The Southern States Communication Association (Winston-Salem,Nc,3-7 April).

Anzai, S., \& Paik, C. (2000). The Development And Validity Investigation Of A Classroom Communication Apprehension Scale For Japans High School Students, National Association Of Hispanic And Latino Studies (Houston,Tx,21-26 February).

Barakat, Z. (2005). The relationship between reflective thinking and academic achievement of undergraduates and high school students in light of some variables. Bhrain: Psychological and Educational Sciences Journal, 6(4).

Dejancuk, N., \& Dejancukova, D. (2003). On Perspectives of Reflective Education. Retrieved from http://www.wcp2003.org/NikolajDemjancuk_Dagmar Demjancukova.doc.2003

Ellison, C. (2008). Reflective Make-and-take: A Talent Quest Reflective Practice Teacher Model. Reflective Practice, 9(2), 185-195.

Farrell, T. (2008). Reflective Practice in the Professional Development of Teachers of Adult English Language Learners. Retrieved from http://www.teslontario.org/uploads/research/Reflective PracticeFinalWeb\%20Farrell.pdf.

Guilford, J. P. (1986). Creative talents; Their nature, Uses and development. NY: BearlyLimited Buffalo.

Herrman, N. (2002). The Creative Brain. Retrieved from https://criticalthinking156.wikispaces.com /file/view/Creative+Brain+Hermann.pdf

Ibrahim, M. A. (2005). Thinking from an educational perspective. Egypt: Cairo, the world of 
books.

khawlda, A. (2012). Linguistic evaluation for writing and reflective thinking. Amman: Alhamd for publishing and distribution.

Kim, Y. (2005). Cultivating Reflective Thinking : The Effects of a Reflective Thinking Tool on Learners' Learning Performance and Metacognitive Awareness in the Context of On-Line Learning. Unpublished Doctoral Dissertation, The Pennsylvania State University.

Mohammed, S. K. (1993). Reflective Thinking: a method of Education. Cairo: Arab Renaissance Publishing House Printing and Publishing.

NCTM. NCATE/NCTM. (2003). Program Standards, Standards for Middle Level Mathematics Teachers. Retrieved from http:/www.ncate.org/ProgramStandards/ NCTM/NCTMSECONStandards.pdf

Ogonor, B., \& Badmus, M. (2006). Reflective Teaching Practice Among Student Teachers: The Case in a Tertiary Institution in Nigeria. Australian Journal of Teacher Education, 31(2), $1-11$.

Olaimat, M.(1994). the development of a measure of attitudes towards the teaching profession. Arab Journal of Education, 14.

Scott, S., \& Issa, T. (2007). Closing the Loop: The Relationship Between Instructor Reflective Practices and Student Satisfaction and Quality Outcomes. Retrieved from http://108.cgpublisher.com/proposal/165/index_html

Shaka, A. (2007). level of reflective thinking among under and post graduate students. AlNajah University. Journal of AlNajah University for Research, (4), 21.

Shaw, M. E., \& Wright, J. M. (1967). Scales for the measurement of attitudes. New York: McGraw-Hill.

Shwaheen, K. (2009). The development of thinking skills in learning science. Amman: Dar almaseera for publication and distribution.

Van Hoof, E., De Becker, P., \& De Meirleir, P. (2006). Pediatric chronic fatigue syndrome and Munchausen-by-Proxy: a case study. Journal of Chronic Fatigue Syndrome, 13(2/3), 45-53.

Zahran, H. (1974). Social Psychology, Cairo: the world of books, 1974.

\section{Copyright Disclaimer}

Copyright reserved by the authors.

This article is an open-access article distributed under the terms and conditions of the Creative Commons Attribution license (http://creativecommons.org/licenses/by/3.0/). 